\section{Mandatory Neuroendovascular Evolution: Meeting the New Demands}

\author{
Mohammad El-Ghanem ${ }^{a} \quad$ Francisco E. Gomez $^{\mathrm{b}}$ Prateeka Koul ${ }^{\mathrm{c}}$ \\ Rolla Nuoman ${ }^{d}$ Justin G. Santarellie Krishna Amuluru ${ }^{f}$ \\ Chirag D. Gandhi ${ }^{\mathrm{e}}$ Eric R. Cohen ${ }^{\mathrm{g}}$ Philip Meyers ${ }^{\text {h }}$ Fawaz Al-Mufti ${ }^{\mathrm{e}}$ \\ aDepartment of Neurology, University of Arizona, Tuscon, AZ, USA; ${ }^{b}$ Department of \\ Neurology, University of Pennsylvania, Philadelphia, PA, USA; ' Department of Internal \\ Medicine, Stamford Hospital, Stamford, CT, USA; d Department of Neurology, Rutgers \\ New Jersey Medical School, Newark, NJ, USA; ${ }^{e}$ Department of Neurosurgery, New York

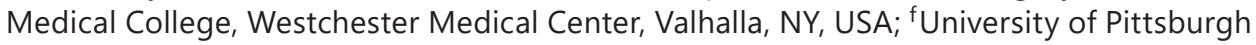 \\ Medical Center Hamot, Great Lakes Neurosurgery and Neurointervention, Erie, PA, USA; \\ 9 Department of Neurology and Neurosurgery, Rutgers Robert Wood Johnson Medical \\ School, Piscataway, NJ, USA; h Department of Radiology, Columbia University Medical \\ Center, New York, NY, USA
}

\section{Keywords}

Stroke - Telestroke - Prehospital stroke scale - Endovascular treatment - Emergent large vessel occlusion - Mechanical thrombectomy

\begin{abstract}
Background: Traditionally, patients undergoing acute ischemic strokes were candidates for mechanical thrombectomy if they were within the 6-h window from onset of symptoms. This timeframe would exclude many patient populations, such as wake-up strokes. However, the most recent clinical trials, DAWN and DEFUSE3, have expanded the window of endovascular treatment for acute ischemic stroke patients to within $24 \mathrm{~h}$ from symptom onset. This expanded window increases the number of potential candidates for endovascular intervention for emergent large vessel occlusions and raises the question of how to efficiently screen and triage this increase of patients. Summary: Abbreviated pre-hospital stroke scales can be used to guide EMS personnel in quickly deciding if a patient is undergoing a stroke. Telestroke networks connect remote hospitals to stroke specialists to improve the transportation time of the patient to a comprehensive stroke center for the appropriate level of care. Mobile stroke units, mobile interventional units, and helistroke reverse the traditional hub-and-spoke model by bringing imaging, tPA, and expertise to the patient. Smartphone applications and social
\end{abstract}


media aid in educating patients and the public regarding acute and long-term stroke care. Key Messages: The DAWN and DEFUSE3 trials have expanded the treatment window for certain acute ischemic stroke patients with mechanical thrombectomy and subsequently have increased the number of potential candidates for endovascular intervention. This expansion brings patient screening and triaging to greater importance, as reducing the time from symptom onset to decision-to-treat and groin puncture can better stroke patient outcomes. Several strategies have been employed to address this issue by reducing the time of symptom onset to decision-to-treat time.

(c) 2018 S. Karger AG, Base

\section{Introduction}

Stroke is the fourth leading cause of death in the United States [1]. Nearly 800,000 strokes occur annually in the United States [2], with the total cost of stroke burden projected from 2005 to 2050 to be USD 2.2 trillion [3]. As the goal for acute stroke treatment is to reperfuse and salvage brain parenchyma, mechanical thrombectomy was developed as a treatment modality for patients unable to undergo the standard intervention of fibrinolysis [4]. Trials such as MR CLEAN and ESCAPE found patients treated with mechanical thrombectomy as opposed to standard care with improved 90-day modified Rankin scores and outcomes, with treatments initiated within $6 \mathrm{~h}$ of symptom onset, setting the time window for endovascular intervention $[5,6]$. However, with the results of the DAWN and DEFUSE3 trials, this window has expanded to $24 \mathrm{~h}$ from symptom onset for certain acute stroke patients [7, 8]. The DAWN trial had a number needed to treat (NNT) of 2.8, an astonishing feat when compared to other life-saving interventions, such as NNT of 8 for thrombolysis in acute ischemic stroke or NNT of 17 for PCI for high-risk STEMI (Fig. 1) [4, 8-10]. This expansion will lead to an increase in the number of stroke patients eligible for endovascular intervention, since patients with wake-up strokes or those a distance away from the nearest comprehensive stroke center may fall within the new window. Whether increasing primary and comprehensive stroke centers or increasing utilization of pre-hospital triaging tools, the anticipated increase in endovascular candidates past the prior 6 -h treatment window need to be appropriately treated. Several strategies have been employed to maximize the efficiency of care to reduce time to diagnosis or treatment and thus ensure better outcomes of acute ischemic stroke patients.

\section{Overview of Strategies and Tools (Fig. 3)}

\section{Stroke Screening by Initial Responders}

The 2018 Guidelines for acute stroke management for health professionals by the American Heart Association and American Stroke Association emphasizes the need for better EMS screening tools for rapid identification and transport of patients to the appropriate level stroke center [11]. Although several smaller-scale studies examining the use of pre-hospital stroke screening tools have shown promising sensitivities and specificities in their respective hospital networks, a larger-scale study with specific standardization of criteria may be warranted to improve pre-hospital stroke screening and outcomes across the United States. For instance, De Luca et al. [12] performed a cross-sectional multicenter study of 38 Italian operative centers (centers that work with EMS personnel in transporting patients to the appropriate emergency department) using the Cincinnati Prehospital Stroke Scale (CPSS) and found of 21,760 cases, the positive predicted value was $34 \%$ and sensitivity was $64 \%$, with higher values of 56 and 71\%, respectively, at centers using CPSS more often. 
Fig. 1. Number needed to treat (NNT) for key medical therapies. Reprinted with permission from Kamal et al. [4].

\begin{tabular}{|l|l|}
\hline Therapy & NNT \\
\hline Decompressive hemicraniectomy for middle cerebral artery infarction & 2 \\
\hline Defibrillation for cardiac arrest & 2.5 \\
\hline Thrombectomy for acute ischemic stroke (DAWN) & 2.8 \\
\hline Goal directed therapy for acute sepsis & 5 \\
\hline Thrombolytis for acute ischemic stroke $<3 \mathrm{~h}$ & 8 \\
\hline Ipatropium and beta-agonist nebulizer for childhood asthma & 11 \\
\hline Thrombolytis for acute ischemic stroke $>3$ h and $<4.5 \mathrm{~h}$ & 15 \\
\hline PCI for high risk ST-elevation myocardial infarction & 17 \\
\hline Aspirin for ST-elevation myocardial infarction & 42 \\
\hline Antibiotics for the primary prevention of rheumatic fever & 53 \\
\hline Bariatric surgery on mortality in obese patients & 77 \\
\hline
\end{tabular}

The ultimate goal for pre-hospital screening is the rapid recognition of large vessel occlusions (LVO) and appropriate routing to a mechanical thrombectomy-capable hospital [13, 14]. Performing a full National Institutes of Health Stroke Scale (NIHSS) may prove cumbersome in the field and may not detect an LVO regardless of level of clinical acumen of the healthcare worker. To combat this issue, several abbreviated stroke scales have been employed in the search for a higher sensitivity, specificity, and convenience. These tools focus on clinical features that are highly suggestive of an LVO, such as gaze deviation, motor weakness, and visuo-spatial neglect [13,15-19]. Some that have shown promising sensitivities and specificities in the literature include: Rapid Arterial Occlusion Evaluation (RACE) scale, Prehospital Acute Stroke Severity scale, Los Angeles Prehospital stroke screen (LAPSS) scale, Field Assessment Stroke Triage for Emergency Destination (FAST-ED) scale, Vision Aphasia Neglect scale, CPSS scale (CPSSS), 3-item stroke scale (3I-SS), and shortened NIHSS for EMS (sNIHSS-EMS).

- 3-Item Stroke Scale (3I-SS): This 3-item scale assesses disturbance of consciousness $(0-2)$, gaze \& head deviation (0-2), hemiparesis (0-2). A cohort of 83 patients were examined with this scale as well as with NIHSS. The 3I-SS showed a strong association with the NIHSS, with a score of $\geq 4$ of proximal vessel occlusion showing a sensitivity of $67 \%$ and specificity of $92 \%$ [20].

- Cincinnati Prehospital Stroke Severity Scale (CPSS): This diluted NIHSS examined gaze (2), arm paresis (1) and level of consciousness (1) for a potential total of 4. A 2015 study found that when CPSSS score $\geq 2$, sensitivity was $89 \%$ and specificity was $73 \%$. This scale was derived based on a study that analyzed which components of the NIHSS were most predictive of severe stroke [15].

- Field Assessment Stroke Triage for Emergency Destination (FAST-ED): Gauges facial $(0-1)$ or arm (0-2) weakness, speech changes (0-2), eye deviation (0-2), and anosognosia/neglect $(0-2)$. This scale was constructed from the NIHSS elements and is considered predictive of ICA and MCA-M1 segment occlusion. The sensitivity of this device is $61 \%$ with a specificity of $89 \%$ [13].

- Prehospital Acute Stroke Severity scale: Evaluates: consciousness (e.g., incorrect month and/or age), gaze deviation and arm weakness. Sensitivity is $66 \%$ with a specificity of 83\%. This device is attuned to the detection of LVOS, with similar NIHSS scores [18].

- $\quad$ Rapid Arterial Occlusion Evaluation (RACE): Evaluates aphasia/agnosia (0-2), facial, arm or leg paresis (0-2 each), gaze deviation (0-1), with a sensitivity of $85 \%$ and specificity 


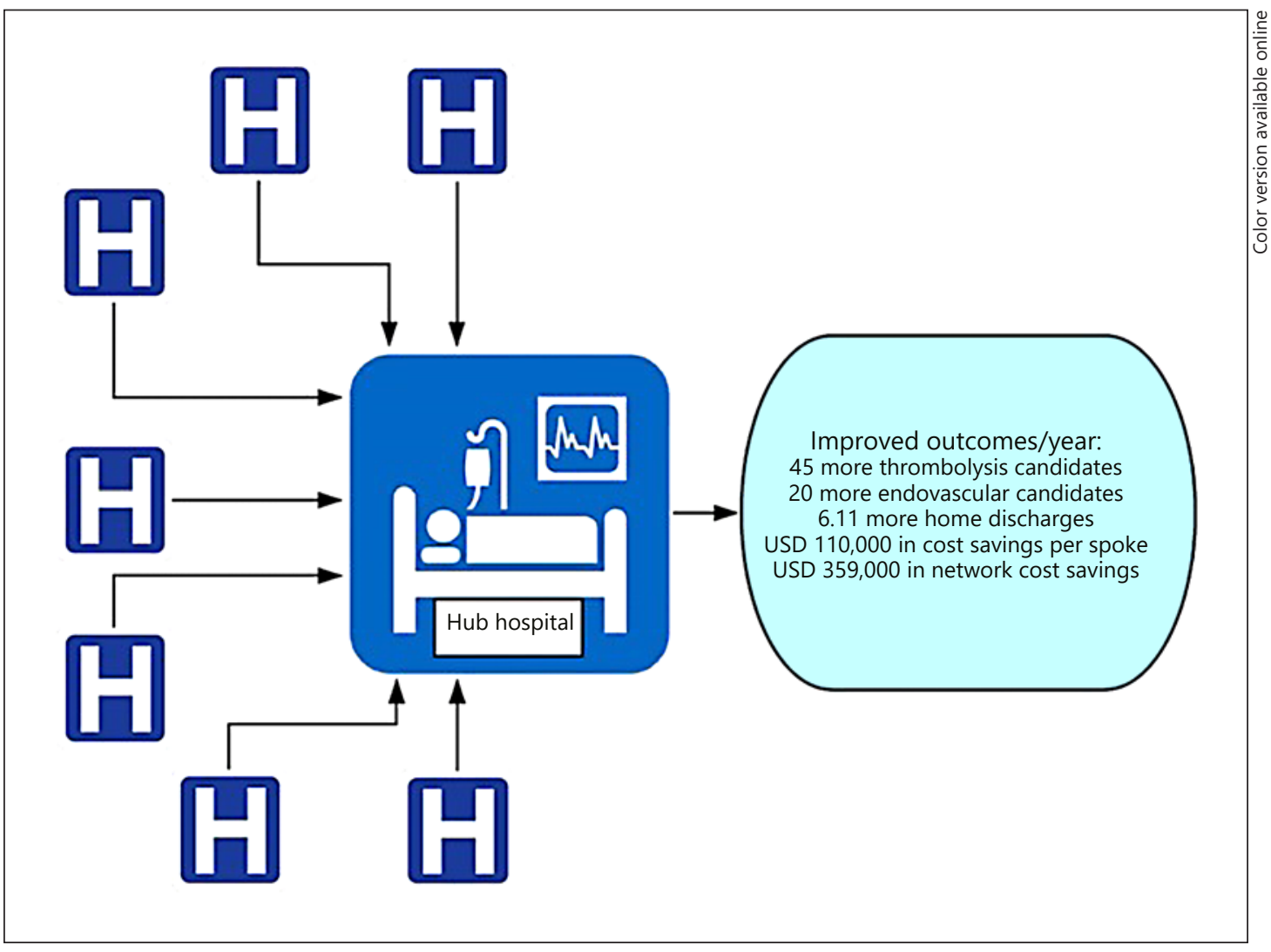

Fig. 2. Hub-and-spoke model.

of $68 \%$ Initial trials showed the RACE scale in the pre-hospital setting by emergency medical technicians were comparable with that of the NIHSS done by a neurologist in a hospital setting [17]. Further attempts to streamline this device lowered predictive value [21].

- $\quad$ Shortened-NIHSS for EMS (sNIHSS-EMS): Evaluates Level of Consciousness (0-3); Facial Palsy (0-3); Left Motor Arm (0-4); Right motor arm (0-4); Left motor leg (0-4); Right motor leg (0-4); Sensory (0-2); Best Language (0-3); Dysarthria (0-2). This scale was designed to mirror the NIHSS, the established gold standard in assessing the severity of acute stroke, as it monitors stroke progression and predicts if the patient is experiencing an LVO. Seven hundred forty-one patients were predicted to have an LVO, with ROC analysis showing optimal cutoff point of a score $\geq 6$, with sensitivity of $70 \%$ and specificity of $81 \%$ [22].

- Vision-Aphasia-Neglect: Evaluates aphasia, neglect, arm paresis, and weakness. This device was designed specifically for MCA evaluation, showing a 100\% sensitivity and $90 \%$ specificity, in a 62-patient cohort [16].

- Volumetric Integral Phase-shift Spectroscopy for Noninvasive Detection of Hemispheric Bioimpedance Asymmetry in Acute Brain Pathology (VITAL). This pioneering new modality for stroke screening would utilize a hand-held visor-like device able to detect cerebral fluid changes in real time without need for special training, as well as differentiate between large and small strokes. Pilot studies showed both sensitivity and specificity in the $90 \%$ range, with large-scale trial findings yet to be published [23]. 


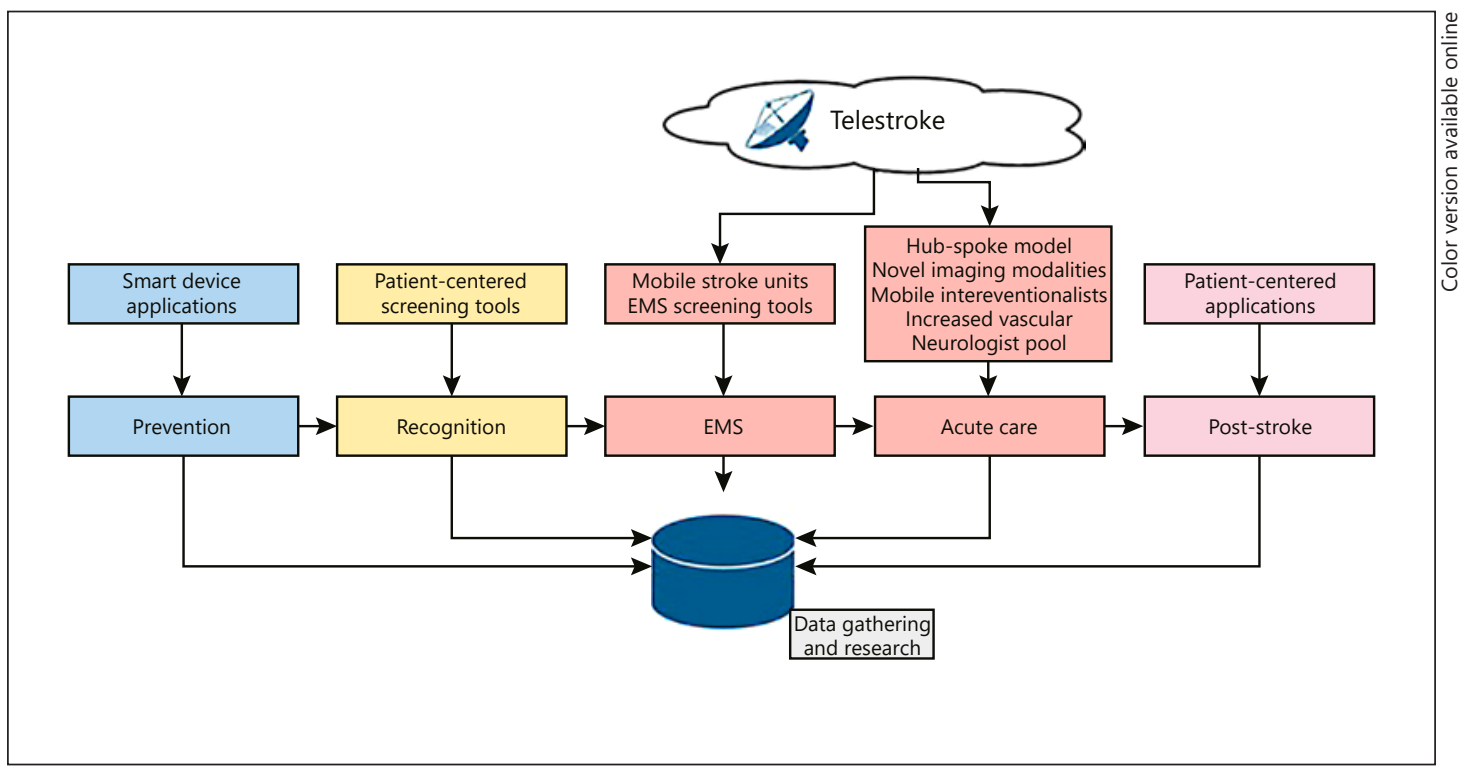

Fig. 3. Applications of novel systems to expand stroke care.

\section{Telestroke}

Advancements in acute stroke care include telestroke technology [24-27]. The concepts of telemedicine and telestroke were pioneered in the 1990s, with the aim of improving care in remote areas or those with limited access to care, such as rural settings [24]. With telestroke, community hospitals without the capabilities to treat an acute stroke can now access the expertise of a stroke neurologist and an endovascular interventionalist to facilitate acute stroke patients faster to the appropriate stroke center for treatment and management. This way, telestroke networks can decrease the burden of stroke at smaller hospitals.

Telestroke systems typically follow a centralized "hub and spoke" design, wherein smaller satellite centers without comprehensive stroke capabilities connect with a stroke specialty hub hospital. When the spoke hospital receives a stroke patient, a consultation with a specialist is triggered at the hub via telestroke network. This model shows an increase in utilized resources and capabilities of the hub as seen in the increase in the number of patients treated with IV tPA and mechanical thrombectomy [28]. Access to endovascular treatment is also enhanced, as candidacy can be rapidly determined and patients can be routed efficiently. In one study, patients transferred to telestroke-equipped centers are more likely to undergo intra-arterial treatment by 11\% (66 vs. 55\%) [27]. Availability of telestroke networks can also facilitate rapid endovascular intervention as advanced notice allows adequate facility and team preparation while the patient is en route [29].

In one model study, the telestroke model was shown to hold several advantages over traditional care. Apart from improving clinical outcomes and costs (Fig. 2), the hub and spoke model proved to be economically feasible for systems comprising more than 2 spoke centers. Although the hub hospital incurred increased costs nearing USD 410,000, the overall savings for the system led to a net positive result [30].

\section{Electronic Aids}

The advent of smartphones and tablets brought an explosion in the field of mobile programs [31]. There are nearly 5,000,000 smartphone applications between the leading smartphone platforms [32], with more than 165,000 of these focused on health [33]. 
Several applications have been developed for primary and secondary prevention purposes. For instance, the Korea University Health Monitoring System for Stroke provides patients that experienced a past stroke with information regarding ongoing preventive vascular risk management [34]. Another example is mHealth, an application designed to aid stroke patients in long-term care such as providing instructions of care and medication management [35]. Artificial intelligence was utilized in an application to predict and combat anticoagulation nonadherence in patients with limited smartphone experience [36].

A number of these programs focus on streamlining EMS operations. Some of these programs do so by including stroke scale calculators, which may tally scores automatically, such as MDCalc or Stroke Pocketcards. Other applications allow real-time encrypted communication between emergency responders and hospital personnel, such as Twiage or Pulsara. These programs allow paramedics to share real-time information with receiving hospitals, including photographs or videos to hospitals to prepare for patient arrival. Applications may also be used to telecast suggestive symptoms to telestroke networks or stroke physicians $[37,38]$.

Numerous other applications have been developed to aid first-line personnel in quickly relaying information to stroke specialists for deciding upon the course of acute treatment and management for patients that likely experienced an acute stroke. For instance, Stroke119 is a comprehensive application with a built-in CPSS-based screening scale that aids in the initial recognition of stroke symptoms [39]. End users select from a set of images that best describes the present symptoms and a stroke probability is calculated. The application also directs users to nearby hospitals with the appropriate stroke capabilities. In addition, there is a hybrid web-portal functionality for hospitals to keep their geographic information upon the application up-to-date through a web browser [39].

Electronic medical records such as Epic may offer remote access and image viewing. Other applications, such as ResolutionMD, may directly route acquired images such as CT scans to telestroke network physicians' smart devices, expediting diagnostic or treatment determinations [40]. In one study, ResolutionMD versus a widely available system, the Picture Archiving and Communication System (PACS), showed very high inter-rater agreement [41]. The use of ResolutionMD as an adjuvant to PACS allows the user to access PACS images upon their smartphones and reduces the amount of time for the stroke specialist to choose appropriate treatment for the patient. Although smartphone display screens may have limitations in the resolution and quality of displaying images, smartphones continue to rapidly advance and have shown good inter-rater agreement when compared to images viewed on a proper medical station desktop $[42,43]$.

\section{Mobile Stroke and Interventional Units}

Despite the implementation of telestroke and other efforts to streamline acute stroke care, the time between symptom onset and treatment of stroke has plateaued around an average of $130 \mathrm{~min}[10,25,44-50]$. The greatest delay in the treatment of stroke patients has been shown to be in the prehospital phase, in which the patient is triaged and transported to a specialized facility [51].

With the goal of decreasing time to treatment, a system that made portable many stroke care capabilities to the patient was devised, thus leading to the creation of the mobile stroke unit (MSU). The MSU is a modified ambulance that contains a CT scanner and healthcare specialists with the authority to determine the patient's treatment and management or seek expertise via the appropriate telestroke network.

The first unit was developed in Berlin and resulted in a decrease in time to diagnosis and treatment in stroke patients $[50,52]$. The Prehospital Acute Neurological Treatment and Optimization of Medical Care in Stroke Study compared MSUs with standard of care [53]. 
Notification to treatment was reduced by $25 \mathrm{~min}$, while patients receiving thrombolysis increased by $12 \%$ with no comparative increase in complications secondary to thrombolysis [45]. Another controlled German study showed MSUs a reduction in notification to therapy time averaging $41 \mathrm{~min}$, with MSUs having a median time of $35 \mathrm{vs.} 76 \mathrm{~min}$ without the MSUs [50].

Neurointerventional-trained specialists are not universally available, with prior estimates putting the number of practitioners at nearly 800 in the United States [54]. Mobile neurointerventional teams were piloted in the Kanazawa Mobile Embolectomy Team conducted in Japan. Local physicians within an $80-\mathrm{km}$ radius would administer tPA as they deemed appropriate and would alert the Kanazawa Mobile Embolectomy Team unit. Results have been promising, with a revascularization rate of $80 \%$ and mean onset to puncture times of $224 \mathrm{~min}$, not dissimilar to those seen in traditional teams [55]. Although the radius for this study was somewhat limited, it did show promise in terms of feasibility for mobile endovascular teams. However, cost effectiveness and applicability remain to be evaluated upon a larger scale.

CT angiography (CTA) capable units could allow endovascular candidates to be rapidly identified on the field, enabling efficient routing of potential thrombectomy candidates to endovascular-capable centers. In one study, this approach nearly halved door-to-puncture time eligible patients (93 vs. $200 \mathrm{~min}$ ) [56]. This study used telestroke to contact the stroke center's vascular neurologist to determine the patient's eligibility for CTA. Having MSUs with this level of neuroimaging would greatly expedite determining a stroke patient's candidacy for endovascular intervention.

Another way to reduce treatment times is to transport a neurointerventionalist to where the patient is having the stroke. Hui et al. [57] designed and implemented a proof-of-concept case to demonstrate the potential cost-savings of transporting a neurointerventionalist via helicopter to the patient in an angiographic-capable hospital. Transporting a patient via helicopter can require advanced levels of care, such as personnel to manage intravenous lines and endotracheal tubes, which can increase the hospital bill for the patient [57]. The hospital in which the patient was admitted had an angiography suite that the authors had supplied with the appropriate catheters and devices; however, there was no neurointerventionalist on site. This case had a total time from last well known to groin closure of $153 \mathrm{~min}$, with a total time from decision to treat to groin closure of $77 \mathrm{~min}$, times competitive with those reported in the literature [58]. However, Friedrich et al. [59] discusses potential problems that may arise from this reverse spoke-and-hub model. For instance, in the case discussed by Hui et al. [57], they had to supply the angiography suite with appropriate equipment. Neurointerventionalists may have to be stocked with the appropriate equipment and travel to angiography suites that may not be amenable for treating patients with mechanical thrombectomy [59].

Some EMS systems utilize "lights and siren" when transporting stroke patients to the hospital $[60,61]$. Transporting an acute ischemic stroke patient to the hospital can otherwise have time delays, which can reduce the chances for brain tissue to be salvageable. Allowing a neurointerventional specialist to utilize "lights and sirens" to reduce the time from patient's last well known to decision to treat could greatly increase the chances of saving a patient's life. For instance, Dr. Kaushal at Palmetto General Hospital uses emergency lights upon his own vehicle to drive past roads with heavy traffic while still abiding by traffic laws [62]. This could be useful in conjunction with the concept of helistroke, especially for regional hospitals that do not have neurointerventional expertise on site.

Although the reduction in notification to treatment times would be expected to improve clinical outcomes, analyses have yielded unchanged patient survival and disability scores at 90 days [45]. Another issue MSUs face is poor cost-effectiveness given the necessary initial investment and high operational costs as compared to the reaped benefits of improved patient 
outcomes. In one analysis of Cleveland-based MSUs, 317 deployments of the units yielded 16 tPA administrations, 4 within $1 \mathrm{~h}$ of initial notification [63]. Nevertheless, MSUs remain a value proposition in rural areas where access to a stroke center is limited [64].

\section{Hospital-Based Strategies}

How can comprehensive stroke centers increase their capabilities of handling increased amounts of patients that may be candidates for mechanical thrombectomy? The shortage of neurovascular specialists is well recognized with only about 1,100 physicians currently available, and stroke incidence projected to outstrip supply [2]. Since 2012, only 10 new ACGME-Recognized Vascular Neurology fellowships have been inaugurated, which totals 82 at the time of this manuscript. The heterogeneous distribution of neurovascular specialists complicates matters, with underserved areas exhibiting the lowest density of vascular neurologists [65-67]. Several strategies have been suggested to meet this need, including increasing the number of vascular neurology training positions, decreasing accumulated tuition burdens, and improving reimbursement for this specialty [2].

\section{Stroke Education in the Setting of Pop Culture}

Much focus has been placed upon educating healthcare personnel like EMS workers to recognize and transport potential stroke patients to the appropriate hospital facility. Another approach to reduce the door to needle time for acute stroke patients is to reduce the time from stroke recognition to activation of the emergency response system [14]. Williams and Noble [68] published a study where the use of hip hop music and dance was used to teach elementary school children in Harlem, NYC to recognize the FAST symptoms of stroke. Since the demographic in central Harlem consists of adults with risk factors for stroke, educating the children this way could increase the spread of knowledge of symptom recognition and prevention to the adults with trouble accessing healthcare [68].

Bundy et al. [69] performed an analysis of a Twitter network geared toward stroke education and care found by searching using the key term \#Stroke from March 20, 2012 to January 31,2018 . They noted that advances in stroke care, especially since the results of the DAWN and DEFUSE3 trials, have increased tweets regarding thrombectomy [69]. Since the upcoming generation of adolescents and adults use social media for communication and information, such media platforms can reach out to and educate millions of laypersons about acute stroke symptom recognition, treatment, and care.

Other forms of social media are also utilized to quickly disperse information relating to stroke awareness and education. For instance, YouTube is a popular platform to view videos with content ranging from entertainment to education. Several healthcare organizations and physicians utilize this to include didactic visuals and audio to explain disease prevention and treatment for various medical conditions like acute stroke. Similarly to Twitter, millions of users access YouTube daily, making this an extremely effective tool to educate the general population in stroke symptom recognition and prevention. Future studies should correlate the effect of the availability of such information to the education level of stroke patients and their family and friends. This could help determine if the content created is understood at a layperson level and engaging enough to attract the attention of the general population.

\section{Novel Imaging Applications}

Stroke centers must possess the necessary imaging modalities to determine endovascular or intravenous treatment candidacy. Thus, expanding image acquisition capabilities should be pursued. CT modalities are more widely available than MRI, with generally shorter acquisition times, and potentially more cost-effective to implement. 
CT Perfusion (CTP) scans have been shown to best depict salvageable tissue for intervention. Another way to approach this is to increase the amount of hospitals with CTP capabilities to potentially decrease the door-to-puncture time for ET candidates [70, 71]. A retrospective cohort study used diffusion-weighted and perfusion-weighted imaging to identify large-vessel occlusions and resulted in $95.9 \%$ sensitivity and $98.4 \%$ specificity, suggesting the utilization of these imaging modalities to identify candidates for endovascular therapy [72]. In addition, cone-beam CT can be used in conjunction with the aforementioned abbreviated stroke scales to reduce door to groin puncture times [73]. Another modality to consider is volume perfusion CT with CTA vessel reconstruction, which was shown to reduce time to puncture in initial trials [74].

Other novel imaging applications include image analytic software. In one trial, an automated image processing software package termed RAPID was employed. This system was able to process perfusion and diffusion weighted MRI images and effectively identify advantageous reperfusion candidates [75]. With the increased window of endovascular treatment from within $6 \mathrm{~h}$ to within $24 \mathrm{~h}$, such imaging software becomes vital for determining candidates for endovascular intervention from the anticipated increase in patients from the DAWN and DEFUSE3 trials [7, 8]. In conjunction with the pre-hospital abbreviated screening scales, automated imaging software and CTP can form the basis of a streamlined process of triaging patients for the appropriate level of care needed.

\section{Research Applications}

Conversion of medical and imaging records to digital form has well served to facilitate data collection analysis and sharing. Several databases have been founded, including the StrokeNet, NIH Imaging Workgroup, and the Stroke Imaging Research/Virtual International Stroke Trials Archive Collaboration. However, problems such as data non-standardization have posed challenges in the creation of easily accessible data repositories [76]. As an example, the StrokeNet consists of 25 regional centers and nearly 200 individual hospitals working in concert to advance cerebrovascular research.

Other applications have been developed to facilitate data gathering while being integrated to stroke care. For example, the Smartphone Assisted Pre-hospital Medical Information System permitted EMS personnel to complete the CPSS on a smart device, while neurovascular specialists could access patient information on the same system [77]. This led to a more effective patient and data management than that was possible with traditional systems [77]. Furthermore, stroke registries allow neurointerventional and stroke specialists to better analyze outcomes [78].

Another incipient technology with potential far-reaching implications is image analytic artificial intelligence [79]. These modalities have been successfully employed in other applications with success and will likely find applications in the near future for neruovascular cases.

\section{Conclusion}

The application of IV-tPA and endovascular therapies has greatly reduced the burden of stroke. However, the time-critical nature of these therapies has limited patient access. DAWN and DEFUSE3 are breakthrough trials in the field of vascular neurology, paving the way for stroke and neurointerventional specialists to increase the number of patients as candidates for endovascular interventions such as mechanical thrombectomy. Thus, comprehensive stroke centers will face an increasing number of patients, and the healthcare system must adapt in the coming years in order to manage these increased needs. Since the DAWN and 
DEFUSE3 trials used patients with LVO, future studies including patients with smaller-scale strokes should be tested with the new window of 6-24 $\mathrm{h}[80,81]$.

Stroke capabilities must grow apace, yet infrastructure growth comes at a cost, which can be reduced by efficient usage of available resources. Furthermore, this brings to the forefront the widespread need of appropriate neuroimaging modalities throughout neurointerventional-capability centers. Although CTP is typically deemed the modality of choice for selecting an endovascular candidate, for centers that may not have this imaging method, CT Angiogram could be a consideration for more widespread distribution and use among many healthcare facilities. Additional studies should be performed comparing CTA and CTP with DWI for patients in the 6-24 h window to determine the potential use for CTA in facilitating endovascular therapy candidate selection in networks unable to provide CTP capabilities [82]. Methodologies such as telestroke networks, agile stroke scales and technology-enhanced care have decreased treatment times and improved outcomes. Other systems, such as MSUs and embolectomy teams, remain to be proven effective but may offer better stroke care access to underserved areas. Ultimately, we must adapt and avail ourselves of all tools possible in the service of our acute ischemic stroke patients.

\section{Acknowledgment}

None.

\section{Statement of Ethics}

The authors have no ethical conflicts to disclose.

\section{Disclosure Statement}

The authors have no conflicts of interest to declare.

\section{Funding Sources}

No sources of funding were utilized for this review.

\section{Author Contributions}

Dr. Mohammad El-Ghanem, Dr. Francisco E. Gomez and Dr. Prateeka Koul contributed to drafting and revising the manuscript. Dr. Rolla Nuoman, Dr. Justin G. Santarelli, Dr. Krishna Amuluru, Dr. Chirag D. Gandhi, Dr. Eric R. Cohen, Dr. Philip Meyers, and Dr. Fawaz Al-Mufti contributed to revising the manuscript.

\section{References}

1 FastStats. 2018.

2 Leira EC, Kaskie B, Froehler MT, Adams HP Jr. The growing shortage of vascular neurologists in the era of health reform: planning is brain! Stroke. 2013 Mar;44(3):822-7.

3 Demaerschalk BM, Hwang HM, Leung G. US cost burden of ischemic stroke: a systematic literature review. Am J Manag Care. 2010 Jul;16(7):525-33.

4 Kamal N, Majmundar N, Damadora N, El-Ghanem M, Nuoman R, Keller IA, et al. Mechanical thrombectomy - is time still brain? The DAWN of a new era. Br J Neurosurg. 2018 Jun;32(3):245-9.

5 Berkhemer OA, Fransen PS, Beumer D, van den Berg LA, Lingsma HF, Yoo AJ, et al.; MR CLEAN Investigators. A randomized trial of intraarterial treatment for acute ischemic stroke. N Engl J Med. 2015 Jan;372(1):11-20. 
6 Goyal M, Demchuk AM, Menon BK, Eesa M, Rempel JL, Thornton J, et al.; ESCAPE Trial Investigators. Randomized assessment of rapid endovascular treatment of ischemic stroke. N Engl J Med. 2015 Mar;372(11):1019-30.

7 Albers GW, Marks MP, Kemp S, Christensen S, Tsai JP, Ortega-Gutierrez S, et al.; DEFUSE 3 Investigators. Thrombectomy for Stroke at 6 to 16 Hours with Selection by Perfusion Imaging. N Engl J Med. 2018 Feb; 378(8):708-18.

8 Nogueira RG, Jadhav AP, Haussen DC, Bonafe A, Budzik RF, Bhuva P, et al.; DAWN Trial Investigators. Thrombectomy 6 to 24 Hours after Stroke with a Mismatch between Deficit and Infarct. N Engl J Med. 2018 Jan; 378(1):11-21.

9 de Boer SP, Barnes EH, Westerhout CM, Simes RJ, Granger CB, Kastrati A, et al. High-risk patients with ST-elevation myocardial infarction derive greatest absolute benefit from primary percutaneous coronary intervention: results from the Primary Coronary Angioplasty Trialist versus thrombolysis (PCAT)-2 collaboration. Am Heart J. 2011 Mar;161(3):500-507.e1.

10 National Institute of Neurological Disorders and Stroke rt-PA Stroke Study Group. Tissue Plasminogen Activator for Acute Ischemic Stroke. N Engl J Med. 1995;333(24):1581-8.

11 Powers WJ, Rabinstein AA, Ackerson T, Adeoye OM, Bambakidis NC, Becker K, et al.; American Heart Association Stroke Council. 2018 Guidelines for the Early Management of Patients With Acute Ischemic Stroke: A Guideline for Healthcare Professionals From the American Heart Association/American Stroke Association. Stroke. 2018 Mar; 49(3):e46-110.

12 De Luca A, Giorgi Rossi P, Villa GF; Stroke group Italian Society pre hospital emergency Services. The use of Cincinnati Prehospital Stroke Scale during telephone dispatch interview increases the accuracy in identifying stroke and transient ischemic attack symptoms. BMC Health Serv Res. 2013 Dec;13(1):513.

13 Lima FO, Silva GS, Furie KL, Frankel MR, Lev MH, Camargo ÉC, et al. Field Assessment Stroke Triage for Emergency Destination: A Simple and Accurate Prehospital Scale to Detect Large Vessel Occlusion Strokes. Stroke. 2016 Aug;47(8):1997-2002.

14 Al-Mufti F, Dancour E, Amuluru K, Prestigiacomo C, Mayer SA, Connolly ES, et al. Neurocritical Care of Emergent Large-Vessel Occlusion: The Era of a New Standard of Care. J Intensive Care Med. 2017 Jul;32(6):373-86.

15 Katz BS, McMullan JT, Sucharew H, Adeoye O, Broderick JP. Design and validation of a prehospital scale to predict stroke severity: Cincinnati Prehospital Stroke Severity Scale. Stroke. 2015 Jun;46(6):1508-12.

16 Teleb MS, Ver Hage A, Carter J, Jayaraman MV, McTaggart RA. Stroke vision, aphasia, neglect (VAN) assessmenta novel emergent large vessel occlusion screening tool: pilot study and comparison with current clinical severity indices. J Neurointerv Surg. 2017;9(2):122-6.

17 Pérez de la Ossa N, Carrera D, Gorchs M, Querol M, Millán M, Gomis M, et al. Design and validation of a prehospital stroke scale to predict large arterial occlusion: the rapid arterial occlusion evaluation scale. Stroke. 2014 Jan;45(1):87-91.

18 Hastrup S, Damgaard D, Johnsen SP, Andersen G. Prehospital Acute Stroke Severity Scale to Predict Large Artery Occlusion: Design and Comparison With Other Scales. Stroke. 2016 Jul;47(7):1772-6.

19 Fischer U, Arnold M, Nedeltchev K, Brekenfeld C, Ballinari P, Remonda L, et al. NIHSS score and arteriographic findings in acute ischemic stroke. Stroke. 2005 Oct;36(10):2121-5.

20 Singer OC, Dvorak F, du Mesnil de Rochemont R, Lanfermann H, Sitzer M, Neumann-Haefelin T. A simple 3-item stroke scale: comparison with the National Institutes of Health Stroke Scale and prediction of middle cerebral artery occlusion. Stroke. 2005 Apr;36(4):773-6.

21 Carrera D, Campbell BC, Cortes J, Gorchs M, Querol M, Jimenez X, et al. Predictive Value of Modifications of the Prehospital Rapid Arterial Occlusion Evaluation Scale for Large Vessel Occlusion in Patients with Acute Stroke. J Stroke Cerebrovasc Dis. 2017;26(1):74-7.

22 Purrucker JC, Härtig F, Richter H, Engelbrecht A, Hartmann J, Auer J, et al. Design and validation of a clinical scale for prehospital stroke recognition, severity grading and prediction of large vessel occlusion: the shortened NIH Stroke Scale for emergency medical services. BMJ Open. 2017 Sep; 7(9):e016893.

23 Kellner CP, Sauvageau E, Snyder KV, Fargen KM, Arthur AS, Turner RD, et al. The VITAL study and overall pooled analysis with the VIPS non-invasive stroke detection device. J Neurointerv Surg. 2018 Nov;10(11):1079-84.

24 Levine SR, Gorman M. "Telestroke" : the application of telemedicine for stroke. Stroke. 1999 Feb;30(2):464-9.

25 Switzer JA, Levine SR, Hess DC. Telestroke 10 years later- 'telestroke 2.0'. Cerebrovasc Dis. 2009;28(4):323-30.

26 Switzer JA, Demaerschalk BM. Overcoming challenges to sustain a telestroke network. J Stroke Cerebrovasc Dis. 2012 Oct;21(7):535-40.

27 Osteraas N, Conners J, Cutting S, Song S, Cherian L, Diebolt E, et al. Telestroke vs Phone Consultation in Stroke Patients Eligible for Intra-Arterial Therapy (P6.033). Neurology. 2016;86(16 Supplement):P6.033.

28 Moynihan B, Davis D, Pereira A, Cloud G, Markus HS. Delivering regional thrombolysis via a hub-and-spoke model. J R Soc Med. 2010 Sep;103(9):363-9.

29 Akbik F, Hirsch JA, Chandra RV, Frei D, Patel AB, Rabinov JD, et al. Telestroke-the promise and the challenge. Part one: growth and current practice. J Neurointerv Surg. 2017 Apr;9(4):357-60.

30 Switzer JA, Demaerschalk BM, Xie J, Fan L, Villa KF, Wu EQ. Cost-effectiveness of hub-and-spoke telestroke networks for the management of acute ischemic stroke from the hospitals' perspectives. Circ Cardiovasc Qual Outcomes. 2013 Jan;6(1):18-26.

31 Munich SA, Tan LA, Nogueira DM, Keigher KM, Chen M, Crowley RW, et al. Mobile Real-time Tracking of Acute Stroke Patients and Instant, Secure Inter-team Communication - the Join App. Neurointervention. 2017 Sep; 12(2):69-76. 
32 Number of apps available in leading app stores as of 1st quarter 2018. Available from: https://www.statista. com/statistics/276623/number-of-apps-available-in-leading-app-stores/

33 Misra S. New report finds more than 165,000 mobile health apps now available, takes close look at characteristics \& use 2015. Available from: https://www.imedicalapps.com/2015/09/ims-health-apps-report/

34 Seo WK, Kang J, Jeon M, Lee K, Lee S, Kim JH, et al. Feasibility of using a mobile application for the monitoring and management of stroke-associated risk factors. J Clin Neurol. 2015 Apr;11(2):142-8.

35 Sarfo FS, Ovbiagele B. Mobile health for stroke: a promising concept for research and practice. mHealth. 2017 Feb;3:4.

36 Labovitz DL, Shafner L, Reyes Gil M, Virmani D, Hanina A. Using Artificial Intelligence to Reduce the Risk of Nonadherence in Patients on Anticoagulation Therapy. Stroke. 2017 May;48(5):1416-9.

37 https://www.pulsara.com/.

38 https://www.twiage.com/.

39 Nam HS, Heo J, Kim J, Kim YD, Song TJ, Park E, et al. Development of smartphone application that aids stroke screening and identifying nearby acute stroke care hospitals. Yonsei Med J. 2014 Jan;55(1):25-9.

40 Pai A. FDA clears ResolutionMD diagnostic imaging app for all image modalities except mammography 2014 Available from: http://www.mobihealthnews.com/31804/fda-clears-resolutionmd-diagnostic-imaging-appfor-all-image-modalities-except-mammography

41 Demaerschalk BM, Vargas JE, Channer DD, Noble BN, Kiernan TE, Gleason EA, et al. Smartphone teleradiology application is successfully incorporated into a telestroke network environment. Stroke. 2012 Nov; 43(11): 3098-101.

42 Channer DC, Demaerschalk BM. Response to letter regarding article, "smartphone teleradiology application is successfully incorporated into a telestroke network environment". Stroke. 2013 Feb;44(2):e12.

43 Mahendra Perera C, Chakrabarti R. Letter by Chakrabarti and Pererato regarding article, "smartphone teleradiology application is successfully incorporated into a telestroke network environment". Stroke. 2013 Feb; 44(2):e11.

44 Audebert HJ, Kukla C, Vatankhah B, Gotzler B, Schenkel J, Hofer S, et al. Comparison of tissue plasminogen activator administration management between Telestroke Network hospitals and academic stroke centers: the Telemedical Pilot Project for Integrative Stroke Care in Bavaria/Germany. Stroke. 2006 Jul;37(7):1822-7.

45 Ebinger M, Winter B, Wendt M, Weber JE, Waldschmidt C, Rozanski M, et al.; STEMO Consortium. Effect of the use of ambulance-based thrombolysis on time to thrombolysis in acute ischemic stroke: a randomized clinical trial. JAMA. 2014 Apr;311(16):1622-31.

46 Hess DC, Wang S, Hamilton W, Lee S, Pardue C, Waller JL, et al. REACH: clinical feasibility of a rural telestroke network. Stroke. 2005 Sep;36(9):2018-20.

47 Hill MD, Buchan AM. Thrombolysis for acute ischemic stroke: results of the Canadian Alteplase for Stroke Effectiveness Study. CMAJ. 2005;172(10):1307-12.

48 Sairanen T, Soinila S, Nikkanen M, Rantanen K, Mustanoja S, Färkkilä M, et al.; Finnish Telestroke Task Force. Two years of Finnish Telestroke: thrombolysis at spokes equal to that at the hub. Neurology. 2011 Mar; 76(13): 1145-52.

49 Schwab S, Vatankhah B, Kukla C, Hauchwitz M, Bogdahn U, Fürst A, et al.; TEMPiS Group. Long-term outcome after thrombolysis in telemedical stroke care. Neurology. 2007 Aug;69(9):898-903.

50 Walter S, Kostopoulos P, Haass A, Keller I, Lesmeister M, Schlechtriemen T, et al. Diagnosis and treatment of patients with stroke in a mobile stroke unit versus in hospital: a randomised controlled trial. Lancet Neurol. 2012 May;11(5):397-404.

51 Kohrmann M, Schellinger PD, Breuer L, Dohrn M, Kuramatsu JB, Blinzler C, et al. Avoiding in hospital delays and eliminating the three-hour effect in thrombolysis for stroke. Int J Stroke. 2011;6(6):493-7.

52 Fassbender K, Walter S, Liu Y, Muehlhauser F, Ragoschke A, Kuehl S, et al. "Mobile stroke unit" for hyperacute stroke treatment. Stroke. 2003 Jun;34(6):e44.

53 Ebinger M, Rozanski M, Waldschmidt C, Weber J, Wendt M, Winter B, et al.; STEMO-Consortium. PHANTOM-S: the prehospital acute neurological therapy and optimization of medical care in stroke patients - study. Int J Stroke. 2012 Jun;7(4):348-53.

54 Zaidat 00, Lazzaro MA, Linfante I, Nguyen T, Janjua N. Demand-supply of neurointerventionalists for endovascular ischemic stroke therapy. Neurology. 2013 Jul;81(3):305-6.

55 Uchiyama N, Misaki K, Mohri M, Kamide T, Hirota Y, Higashi R, et al. Treatment Result in the Initial Stage of Kanazawa Mobile Embolectomy Team for Acute Ischemic Stroke. Neurol Med Chir (Tokyo). 2016 Dec;56(12): 737-44.

56 John S, Stock S, Masaryk T, Bauer A, Cerejo R, Uchino K, et al. Performance of CT Angiography on a Mobile Stroke Treatment Unit: implications for Triage. J Neuroimaging. 2016 Jul;26(4):391-4.

57 Hui FK, El Mekabaty A, Schultz J, Hong K, Horton K, Urrutia V, et al. Helistroke: neurointerventionalist helicopter transport for interventional stroke treatment: proof of concept and rationale. J Neurointerv Surg. 2018 Mar;10(3):225-8.

58 Powers WJ, Derdeyn CP, Biller J, Coffey CS, Hoh BL, Jauch EC, et al.; American Heart Association Stroke Council. 2015 American Heart Association/American Stroke Association Focused Update of the 2013 Guidelines for the Early Management of Patients With Acute Ischemic Stroke Regarding Endovascular Treatment: A Guideline for Healthcare Professionals From the American Heart Association/American Stroke Association. Stroke. 2015 Oct;46(10):3020-35. 
59 Friedrich B, Maegerlein C, Lobsien D, Mönch S, Berndt M, Hedderich D, et al. Endovascular Stroke Treatment on Single-Plane vs. Bi-Plane Angiography Suites : Technical Considerations and Evaluation of Treatment Success. Clin Neuroradiol. 2018 Jan; https://doi.org/10.1007/s00062-017-0655-z.

60 Kodankandath TV, Wright P, Power PM, De Geronimo M, Libman RB, Kwiatkowski T, et al. Improving Transfer Times for Acute Ischemic Stroke Patients to a Comprehensive Stroke Center. J Stroke Cerebrovasc Dis. 2017; 26(1):192-5.

61 Simonsen SA, Andresen M, Michelsen L, Viereck S, Lippert FK, Iversen HK. Evaluation of pre-hospital transport time of stroke patients to thrombolytic treatment. Scand J Trauma Resusc Emerg Med. 2014;22:65.

62 News C. Florida neurosurgeon uses emergency lights to rush to stroke patients [Online]. 2016. Available from: https://www.cbsnews.com/news/florida-neurosurgeon-stroke-victims-emergency-lights-personal-cartreat-patients-faster/

63 Taqui A, Cerejo R, Itrat A, Briggs FB, Reimer AP, Winners S, et al.; Cleveland Pre-Hospital Acute Stroke Treatment (PHAST) Group. Reduction in time to treatment in prehospital telemedicine evaluation and thrombolysis. Neurology. 2017 Apr;88(14):1305-12.

64 Southerland AM, Brandler ES. The cost-efficiency of mobile stroke units: where the rubber meets the road. Neurology. 2017 Apr;88(14):1300-1.

65 Adornato BT, Drogan O, Thoresen P, Coleman M, Henderson VW, Henry KA, et al. The practice of neurology, 2000-2010: report of the AAN Member Research Subcommittee. Neurology. 2011 Nov;77(21):1921-8.

66 Dall TM, Storm MV, Chakrabarti R, Drogan O, Keran CM, Donofrio PD, et al. Supply and demand analysis of the current and future US neurology workforce. Neurology. 2013;81(5):470-8.

67 Gillum RF, Ingram DD. Relation between residence in the southeast region of the United States and stroke incidence. The NHANES I Epidemiologic Followup Study. Am J Epidemiol. 1996 Oct;144(7):665-73.

68 Williams 0, Noble JM. 'Hip-hop' stroke: a stroke educational program for elementary school children living in a high-risk community. Stroke. 2008 0ct;39(10):2809-16.

69 Bundy JJ, Chick JF, Hage AN, Srinivasa RN, Chaudhary N, Srinivasa RN, et al. \#Stroke. J Neurointerv Surg. 2018 Dec;10(12):e33.

70 Aghaebrahim A, Sauvageau E, Aguilar-Salinas P, Cortez G, Santos R, Hanel RA. Referral facility CT perfusion prior to inter-facility transfer in patients undergoing mechanical thrombectomy. J Neurointerv Surg. 2018 Sep;10(9):818-22.

71 Aguilar-Salinas P, Aghaebrahim A, Cortez GM, Sauvageau E, Hanel R. Abstract WMP5: Triage to a Comprehensive Stroke Center Based on CT Perfusion Imaging versus No CT Perfusion Imaging at the Spoke Hospital. Stroke. 2018;49(Suppl 1):AWMP5-AWMP.

72 Wolman DN, Iv M, Wintermark M, Zaharchuk G, Marks MP, Do HM, et al. Can diffusion- and perfusion-weighted imaging alone accurately triage anterior circulation acute ischemic stroke patients to endovascular therapy? J Neurointerv Surg. 2018 Dec;10(12):1132-1136

73 Amano T, Sato M, Matsumaru Y, Sakuma H, Yoda S, Hamada Y. Intra-arterial Contrasted Cone-beam Computed Tomography Assessment of Vessels Distal from Occluded Site in Acute Ischemic Stroke with Major Vessel Occlusion. Neurol Med Chir (Tokyo). 2017 Jun;57(6):292-8.

74 Yi HJ, Sung JH, Lee DH, Yang SH, Hong JT. A useful diagnostic method to reduce the in-hospital time delay for mechanical thrombectomy: volume perfusion computed tomography with added vessel reconstruction. J Neurosurg. 2018 Apr;1-8.

75 Lansberg MG, Lee J, Christensen S, Straka M, De Silva DA, Mlynash M, et al. RAPID automated patient selection for reperfusion therapy: a pooled analysis of the Echoplanar Imaging Thrombolytic Evaluation Trial (EPITHET) and the Diffusion and Perfusion Imaging Evaluation for Understanding Stroke Evolution (DEFUSE) Study. Stroke. 2011 Jun;42(6):1608-14.

76 Liebeskind DS, Albers GW, Crawford K, Derdeyn CP, George MS, Palesch YY, et al. Imaging in StrokeNet: Realizing the Potential of Big Data. Stroke. 2015 Jul;46(7):2000-6.

77 Nakae T, Kataoka H, Kuwata S, Iihara K. Smartphone-assisted prehospital medical information system for analyzing data on prehospital stroke care. Stroke. 2014 May;45(5):1501-4.

78 Cadilhac DA, Kim J, Lannin NA, Kapral MK, Schwamm LH, Dennis MS, et al. National stroke registries for monitoring and improving the quality of hospital care: A systematic review. Int J Stroke. 2016 Jan;11(1):28-40.

79 El-Dahshan ES, Hosny T, Salem AB. Hybrid intelligent techniques for MRI brain images classification. Digit Signal Process. 2010;20(2):433-41.

80 Tahsili-Fahadan P. Blogging Stroke [Internet]2018. Available from: https://journals.heart.org/bloggingstroke/2018/02/26/extended-thrombectomy-window-after-stroke-review-of-the-dawn-trial/

81 Gandhi CD, Al Mufti F, Singh IP, Abruzzo T, Albani B, Ansari SA, et al.; Standards and Guidelines committee of the Society of NeuroInterventional Surgery (SNIS). Neuroendovascular management of emergent large vessel occlusion: update on the technical aspects and standards of practice by the Standards and Guidelines Committee of the Society of NeuroInterventional Surgery. J Neurointerv Surg. 2018 Mar;10(3):315-20.

82 Saposnik G, Strbian D. Enlightenment and Challenges Offered by DAWN Trial (DWI or CTP Assessment With Clinical Mismatch in the Triage of Wake Up and Late Presenting Strokes Undergoing Neurointervention With Trevo). Stroke. 2018 Feb;49(2):498-500. 